\title{
INTERVENÇÕES EM HABILIDADES DE VIDA: CONTRIBUIÇÕES PARA O DESENVOLVIMENTO PSICOSSOCIAL DE CRIANÇAS E ADOLESCENTES
}

\author{
INTERVENCIONES EN HABILIDADES PARA LA VIDA: CONTRIBUCIONES AL \\ DESARROLLO PSICOSOCIAL DE NIÑOS Y ADOLESCENTES
}

\section{LIFE SKILLS INTERVENTIONS: CONTRIBUTIONS TO THE PSYCHOSOCIAL DEVELOPMENT OF CHILDREN AND ADOLESCENTS}

Kathyllen Bezerra dos SANTOS ${ }^{1}$ Ana Lúcia Novais CARVALHO ${ }^{2}$

RESUMO: Intervenções direcionadas a crianças e adolescentes que promovam o desenvolvimento psicossocial saudável são relevantes. Nessa perspectiva, destacam-se as que têm como proposta o desenvolvimento das Habilidades de Vida (HV), que são competências definidas pela Organização Mundial da Saúde como fundamentais para melhor lidar com os desafios da vida cotidiana, sendo aliadas na promoção da saúde integral dos indivíduos. Desta forma, o presente estudo teve como objetivo descrever intervenções, em grupo, para o desenvolvimento de HV em crianças e adolescentes realizadas no Brasil. Foi feito um estudo exploratório considerando apenas publicações em língua portuguesa. Foram identificadas oito intervenções, nas quais a idade dos participantes variava dos oito aos dezoito anos. Os principais resultados observados foram a redução de crenças sexistas e homofóbicas, mudanças positivas de comportamento, contribuição para o autoconhecimento e melhoria no relacionamento interpessoal. Conclui-se que tais intervenções são importantes ao impactarem positivamente na qualidade de vida como um todo.

PALAVRAS-CHAVE: Habilidades de vida. Crianças. Adolescentes. Intervenções grupais. Desenvolvimento psicossocial. Promoção de saúde.

RESUMEN: Las intervenciones dirigidas a niños y adolescentes que promueven un desarrollo psicosocial saludable son relevantes. Desde esta perspectiva, destacamos las propuestas para el desarrollo de las Habilidades para la Vida (HV), que son competencias definidas por la Organización Mundial de la Salud como fundamentales para afrontar mejor los retos de la vida cotidiana, y son aliadas para promover la salud integral de los individuos. Así, el presente estudio tuvo como objetivo describir las intervenciones grupales para el desarrollo de la $H V$ en niños y adolescentes realizadas en Brasil. Se realizó un estudio exploratorio considerando sólo las publicaciones en portugués. Se identificaron ocho intervenciones, en las que la edad de los participantes oscilaba entre los ocho y los dieciocho años. Los principales resultados observados fueron la reducción de las creencias sexistas y homófobas, los cambios positivos en el comportamiento, la contribución al autoconocimiento

1 Universidade Federal Fluminense (UFF), Goytacazes - RJ - Brasil. Graduada em Psicologia. ORCID: https://orcid.org/0000-0001-6970-6421. E-mail: kathyllenbezerra@id.uff.br

${ }^{2}$ Universidade Federal Fluminense (UFF), Goytacazes - RJ - Brasil. Professora Associada do Departamento de Psicologia. Doutorado em Biociencias Nucleares (UERJ). ORCID: https://orcid.org/0000-0002-4808-8449. Email: analucianovaiscarvalho@id.uff.br 
y la mejora de las relaciones interpersonales. Concluimos que estas intervenciones son importantes porque tienen un impacto positivo en la calidad de vida en su conjunto.

PALABRAS CLAVE: Habilidades para la vida. Niños. Adolescentes. Intervenciones grupales. Desarrollo psicossocial. Promoción de la salud.

ABSTRACT: Interventions aimed at children and adolescents that promote healthy psychosocial development are relevant. From this perspective, we highlight those proposed for the development of Life Skills (LS), which are competencies defined by the World Health Organization as fundamental for better dealing with the challenges of daily life, and are allies in promoting the integral health of individuals. Thus, the present study aimed to describe group interventions for the development of LS in children and adolescents carried out in Brazil. An exploratory study was carried out considering only publications in Portuguese. Eight interventions were identified, in which the age of the participants ranged from eight to eighteen years old. The main results observed were the reduction of sexist and homophobic beliefs, positive changes in behavior, contribution to self-knowledge and improvement in interpersonal relationships. We conclude that such interventions are important in positively impacting quality of life as a whole.

KEYWORDS: Life skills. Children. Adolescents. Group interventions. Psychosocial development. Health promotion.

\section{Introdução}

Intervenções com o objetivo de diminuir a frequência de comportamentos considerados socialmente inadequados, como a agressividade, apresentados por crianças e adolescentes são amplamente realizados, como trazem as autoras Bolsoni-Silva et al. (2010).

A contribuição para o desenvolvimento integral da saúde de crianças e adolescentes pode se dar a partir de intervenções em Habilidade de Vida (HV), que envolvem habilidades sociais, cognitivas e afetivas (MURTA; DEL PRETTE; DEL PRETTE, 2010) que, como destacado pela Organização Mundial da Saúde (OMS) (1997), auxiliam no lidar com as dificuldades que surgem no cotidiano, promovendo competências psicossociais. Diversos estudos apontam resultados positivos na redução de comportamentos de risco e aumento em competências direcionadas à saúde entre crianças e adolescentes em programas com esta proposta (BOTVIN, 1998; GORAYEB; NETTO; BUGLIANI, 2003; MANGRULKAR; WHITMAN; POSNER, 2001; MINTO et al., 2006).

A Organização Mundial da Saúde (OMS, 1997) compreende que as HV são repertórios comportamentais capazes de promover pessoas mais capazes de lidar com os desafios e vivências que surgem no cotidiano. Desta forma, elas estimulam e promovem a competência psicossocial, auxiliando no bem-estar, seja ele físico, mental ou mesmo social 
dos indivíduos (OMS, 1997), por meio de habilidades interpessoais (OMS, 2003). Ainda de acordo com a OMS (1997), são dez as competências listadas, sendo estas divididas em cinco áreas básicas: tomada de decisão e resolução de problemas; pensamento criativo e pensamento crítico; comunicação e habilidades interpessoais; autoconhecimento e empatia; por fim, lidando com as emoções e lidando com estresse (OMS, 1999).

Atualmente, como trazem Cunha e Rodrigues (2010), são diversos os desafios cotidianos encontrados que podem gerar níveis elevados de estresse, o que afeta muito os sujeitos, independentemente de sua faixa etária. No intuito de lidar com tais questões que demandam uma flexibilidade cognitiva mais expressiva, é necessário tanto o enfrentamento das situações geradoras de estresse, quanto o desenvolvimento de habilidades para tal fim.

A temática se torna ainda mais relevante ao se pensar no enfrentamento da pandemia da COVID-19. Este evento atípico e estressor fez com que as pessoas se confinassem em seus lares, aliado ao medo de contrair o vírus. Em média, um terço das pessoas atravessadas por tal evento, caso não se intervenha logo e corretamente, como trazem dados da Fundação Oswaldo Cruz (FIOCRUZ) (2020a), podem ter manifestações no âmbito psicológico, em que as crianças e adolescentes são também impactadas.

Apesar de a taxa de mortalidade não ser tão expressiva nessa faixa etária, a pandemia muito impactou a rotina da vida de crianças e adolescentes, como com o fechamento de escolas, aumentando expressivamente o tempo que passam em casa, aliado à perda do convívio social (FIOCRUZ, 2020b). Muitas são as reações esperadas e apresentadas pelas crianças ao longo do período pandêmico, tais como "dificuldades de concentração, irritabilidade, medo, inquietação, tédio, sensação de solidão, alterações no padrão de sono e alimentação" (FIOCRUZ, 2020b, p. 4). De tal maneira, os efeitos indiretos da pandemia podem ser tão nocivos quanto os diretos, uma vez que há prejuízos associados à educação formal e à socialização, por exemplo.

Nesse contexto, com a presença de diversas variáveis estressoras, o desenvolvimento de HV pode auxiliar. Tal hipótese se dá, pois, de acordo com a OMS (1997), o ensino de HV amplia a competência psicossocial, auxiliando na promoção de saúde. Como trazem Cunha e Rodrigues (2010), tal competência se mostra relevante ao promover o desenvolvimento de características pessoais que tenham um cunho positivo e mais adaptativo, sendo capaz de promover a saúde psicológica através de recursos, como a resiliência.

Nessa perspectiva, intervenções precoces com o objetivo de desenvolver HV com crianças e adolescentes são cruciais dado que seus estilos de vida, seus hábitos, dentre outros aspectos de sua personalidade, ainda se encontram em constante processo de transformação e 
desenvolvimento. Desta forma, se nota que o público infantojuvenil é mais receptivo, flexível e suscetível a mudanças (RODRIGUES et al., 2008).

Murta, Del Prette e Del Prette (2010) apontam como treinamentos em habilidades sociais, assim como as de vida, podem ser importantes para prevenir sexismo e heterossexismo. Espera-se, também, que a partir do uso de tais competências, adolescentes possam, por exemplo, evitar a gravidez na adolescência, desenvolvendo um comportamento sexual mais seguro, prevenindo infecções sexualmente transmissíveis (IST) ${ }^{3}$, como traz a Organização das Nações Unidas (ONU) (2006) e o HIV/AIDS, como trabalhado na Tailândia e Zimbábue (OMS, 1999). Estudos também apontam que o desenvolvimento das HV potencializa a promoção de autoestima e autoconfiança, prevenindo o bullying (MARYAM et al., 2011; SUPARP, 2010).

Como explicita Botvin e Griffin (2004), as HV podem atuar enquanto estratégia para a redução de comportamentos de risco, promovendo cuidados não só com a saúde física, como também aumento do bem-estar psicológico, atuando, portanto, como fatores de proteção ao uso de substâncias psicoativas entre jovens. Isso denota sua grande valia em intervenções que envolvam crianças e adolescentes no intuito da promoção de saúde e de seu desenvolvimento psicossocial.

Desta forma, o presente estudo tem como objetivo identificar intervenções direcionadas a crianças e adolescentes para o desenvolvimento de HV realizadas no Brasil, identificando suas características, estratégias e resultados. Assim, foi realizado um estudo exploratório, realizado a partir de uma pesquisa bibliográfica.

Com exceção de Neufeld et al. (2014), cujo trabalho fomentou o interesse de buscar mais publicações acerca da temática, fazendo surgir esta pesquisa e foi na mesma incluído, foram buscadas publicações na base de dados Google Scholar, por sua amplitude, utilizando os seguintes descritores de busca: habilidades de vida, habilidades de vida e crianças, habilidades de vida e adolescentes, habilidades para vida e crianças, habilidades para vida e adolescentes, fazendo uso do operador booleano AND entre os descritores. A coleta de dados se deu em junho do ano de 2021, em que os critérios de inclusão foram trabalhos na língua portuguesa, ocorridos no Brasil, com crianças e/ou adolescentes, e que trouxessem o conceito de HV promulgado pela OMS (1997). O período selecionado para a coleta abrangeu publicações realizadas nos últimos 20 anos. Assim, ao final foram identificadas oito intervenções que cumpriam os requisitos da pesquisa.

${ }^{3}$ No arquivo original se utilizou o termo doença sexualmente transmissível (DST), atualmente em desuso, portanto, se substituiu por infecções sexualmente transmissíveis (IST).

Doxa: Rev. Bras. Psico. e Educ., Araraquara, v. 22, n. 00, e021015, $2021 . \quad$ e-ISSN: 2594-8385 


\section{Intervenções em HV com crianças e adolescentes no Brasil}

Uma intervenção com adolescentes foi realizada entre 2001 e 2003 pelo Programa de Promoção de Saúde na Comunidade do Hospital das Clínicas da Faculdade de Medicina de Ribeirão Preto da Universidade de São Paulo (HCFMRP/USP). Ela se deu em uma escola estadual por Minto et al. (2006) e teve como objetivo a promoção de saúde, buscando impactar positivamente na qualidade de vida dos estudantes. Foram 12 sessões com duração média de duas horas cada. Todas as HV foram abordadas, sendo trabalhada uma por sessão, em que se descreve quais técnicas foram utilizadas para desenvolver as habilidades. Concluíram que a intervenção foi adequada, sendo perceptível através de relatos dos participantes, que auxiliou no autoconhecimento, reflexão e no relacionamento interpessoal. Assim, corroborou com os dados descritos na literatura, visto que propiciou o desenvolvimento de habilidades que auxiliam em questões surgidas no cotidiano.

Em sua dissertação de mestrado, Minto (2005) relata a experiência em um programa de $\mathrm{HV}$, durante os anos de 2003 e 2004, para a promoção da saúde e prevenção de comportamentos de risco, como beber, fumar e comportamentos sexuais sem uso de proteção, entre adolescentes de 14 e 17 anos. A intervenção ocorreu em duas instituições, também em Ribeirão Preto. Grande parte dos participantes, dentre os 45 do projeto, era do sexo masculino. $\mathrm{Na}$ análise quantitativa não houve diferenças significativas antes e após a intervenção, em que o ato de beber e fumar estavam acima da média nacional. Contudo, na análise qualitativa foram identificados resultados positivos em que $\mathrm{HV}$ relacionadas ao autocontrole auxiliavam a lidar com situações estressantes envolvendo o contexto familiar, escolar e social.

Murta et al. (2009) trazem em seu artigo resultados positivos de um programa de delineamento pré-experimental em HV com dezoito adolescentes entre 12 e 14 anos, sendo a maioria do sexo feminino. Foram dezesseis sessões realizadas em uma universidade, com duração de aproximadamente 90 minutos, ao longo de cinco meses. Junto às HV foram trabalhadas também outras temáticas, como riscos à saúde associados ao gênero, rede de apoio social e prevenção de recaída, assim como técnicas de treinamento em Habilidades sociais e da Terapia Cognitivo-comportamental (TCC), através de vivências em grupo. A efetividade da intervenção foi positiva, o que se deu a partir do discernimento dos adolescentes e seus cuidadores. Grande parte das metas intermediárias foram alcançadas, dado que havia satisfação por parte dos participantes com os encontros, assim como a prática de habilidades. 
Murta et al. (2009) relatam que todos os participantes se mostraram contemplados com o programa, dado que um ano após a finalização da intervenção relataram não terem tido contato com uso de substâncias lícitas ou ilícitas, IST ou gravidez, em que também se apontou melhoras positivas nas relações com familiares e um maior direcionamento na elaboração de projetos futuros. Houve piora no desempenho escolar de três alunos, todavia, seria necessária uma investigação detalhada para melhor entender tal acontecimento. Os resultados gerais apontam para uma constituição relevante de fatores de proteção que podem auxiliar a lidar com os desafios vivenciados em suas vidas.

Demonstrando como são múltiplas as temáticas que podem ser trabalhadas com $\mathrm{HV}$, Murta et al. (2012) relatam a experiência de um programa que visava promover habilidades interpessoais, direitos sexuais e reprodutivos para adolescentes. Os participantes foram 93 adolescentes, do sexo masculino e feminino, entre 12 e 17 anos de uma escola pública de ensino fundamental em Goiânia. Foram realizadas em 15 sessões que duravam, em média, 90 minutos. A partir de temáticas como violência no namoro, violência contra a mulher e recursos para autoproteção, eram trabalhadas HV tais como relacionamento interpessoal, empatia, lidar com o estresse e a resolução de problemas. Apesar de pontuarem mudanças que seriam relevantes de serem realizadas no intuito de um melhor aproveitamento do programa, como alterações na ordem das sessões, e a inclusão de medidas escritas de avaliação em detrimento a modalidade oral, destaca-se também aspectos positivos da experiência. Isso se dá uma vez que esteve presente uma aceitabilidade entre os adolescentes, isto é, estavam receptivos a discussão dos temas propostos, aspecto de grande importância para o sucesso de uma intervenção. Apontam também como houve aceitação para tratar dos tópicos relativos à sexualidade, gênero e direitos sexuais, gerando engajamento significativo, diferente do apontado na literatura.

Em um outro estudo, Murta et al. (2013) utilizaram as HV para trabalharem junto a adolescentes a prevenção de violência em relacionamentos amorosos, como o namoro, assim como crenças sexistas e homofóbicas. Trata-se de um estudo quase-experimental com 60 alunos de uma escola pública em Brasília, com idades entre 14 e 18 anos. Foi também realizada uma entrevista, cinco meses após a intervenção. Notou-se que, dentre aqueles que participaram do programa, houve mudanças positivas no que diz respeito a respostas sexistas e homofóbicas, havendo diminuição em respostas de tal cunho. No entanto, não houve diferença estaticamente significativa na intenção de enfrentamento à violência no namoro no grupo que passou pela intervenção, em relação ao grupo que não foi exposto, em que se notou 
crescimento na negociação, como para a forma como se estabelece o relacionamento, tanto de forma afetiva quanto sexual. Notou-se também diminuição da resignação.

O Programa Cognitivo-Comportamental de Habilidades de Vida para Crianças e Adolescentes (PRHAVIDA) é uma intervenção baseada em HV feita no Brasil e que ainda se encontra em atividade, desenvolvida por Neufeld et al. (2014), sendo voltada para crianças e adolescentes alunos de uma escola municipal no interior de São Paulo. A intervenção relatada se deu com crianças entre oito e nove anos e adolescentes entre 12 e 13 anos. Os participantes tinham escores heterogêneos em habilidades sociais, depressão, estresse e ansiedade, o que demonstra um olhar para a saúde mental dos participantes, que pertenciam tanto ao sexo masculino quanto feminino.

O programa de Neufeld et al. (2014) foi estruturado em 11 sessões semanais em grupo, com intervenções pré e pós teste. Para além do trabalho de todas as HV, foi também incluído o desenvolvimento de Habilidades Sociais. Os resultados foram considerados positivos, com os participantes relatando o apreço pelo grupo em sessões de avaliação qualitativa ao final. As relações dos participantes do programa entre si melhoraram com o passar das sessões, assim como foi perceptível uma mudança positiva de comportamento por parte dos próprios participantes e terapeutas.

Franco (2014) desenvolveu um estudo de delineamento quase experimental para o desenvolvimento de HV e Habilidades Sociais junto a adolescentes do sexo masculino, em vulnerabilidade social. A média de idade dos participantes era de 12,8 anos e frequentavam uma instituição filantrópica no estado de Minas Gerais. Foram 12 encontros semanais com duração de 60 minutos cada, em que os resultados foram positivos, visto que adquiriram, por exemplo, comportamentos positivos nas interações sociais, em que as HV mais destacadas foram relacionamento interpessoal, comunicação eficaz, tomada de decisão, empatia e pensamento criativo, por serem evidenciadas pelos adolescentes como as mais praticadas por eles em seu cotidiano. Houve também efeitos indiretos no bem-estar dos jovens e direto na habilidade social de civilidade.

Mais recentemente e indo ao encontro ao observado na literatura, como em Paiva e Rodrigues (2008), Nadaleti et al. (2018) apresentaram um estudo quase-experimental que abordava o modelo das HV como prevenção ao uso de substâncias psicoativas entre adolescentes. A intervenção foi realizada em uma escola pública no estado de Minas Gerais, com 78 participantes com idades entre dez e 15 anos. Nadaleti et al. (2018) concluíram que a aplicação do programa foi adequada, auxiliando, por exemplo, no aumento no reconhecimento de fatores de risco e atuando no desenvolvimento de habilidades dos 
adolescentes, apesar da limitação apontada referente ao número reduzido de participantes no estudo.

Foi possível observar que as intervenções aqui relatadas possuem aspectos que se assemelham. Apesar da idade dos participantes ir dos oito aos 18 anos, a grande maioria, com exceção de Neufeld et al. (2014), era voltada para o público adolescente. Por hipótese, acredita-se que tal característica deve ocorrer frente a possíveis dificuldades encontradas ao desenvolver a temática de $\mathrm{HV}$ com públicos mais jovens. Isso se dá, pois, o trabalho com crianças acaba por demandar mais dos aplicadores, por exigir que aspectos lúdicos estejam mais presentes. Percebe-se, também, que os temas escolhidos para as pesquisas se direcionaram às experiências mais concebidas durante a adolescência, como o uso de substância psicoativas, por Nadaleti et al. (2018), e a promoção de direitos sexuais e reprodutivos, por Murta et al. (2012), o que explicaria o foco em determinada faixa etária.

Todas as intervenções ocorreram em grupo, visto que a experiência grupal possibilita, além de atingir diversas pessoas simultaneamente, que muitas das habilidades discutidas sejam colocadas em prática pela troca entre os participantes. O tempo de duração mínimo observado foi de 60 minutos, indo a até 120 minutos, em que metade das intervenções, sendo de Murta et al. (2009), Murta et al. (2013), Neufeld et al. (2014) e Franco (2014), incluíram sessões após a finalização das vivências grupais. Essa coleta de dados se dava nos programas como um todo, através de instrumentos qualitativos, como entrevistas.

Por diversas vezes as temáticas trabalhadas não se limitavam às $\mathrm{HV}$, incluindo também Habilidades Sociais, como visto em Murta et al. (2009), Murta et al. (2012), Neufeld et al. (2014) e Franco (2014). Muitos dos trabalhos versavam sobre temáticas distintas, algo que demonstra a grande valia das intervenções envolvendo as $\mathrm{HV}$, visto que como pontuado anteriormente, é capaz de ser útil para diferentes objetivos. Todavia, é importante salientar que todas abordavam, direta ou indiretamente, a questão referente à promoção de saúde.

A partir do demonstrado, destaca-se a relevância que as intervenções em HV possuem, dado que seus pressupostos podem se adequar às mais diversas demandas, tendo resultados benéficos. Aliado a isso, é notável seu impacto positivo no desenvolvimento psicossocial de crianças e adolescentes, tal qual observado no Brasil, mesmo frente a quantidade ainda não muito expressiva de publicações tratando da temática, o que denota a necessidade de mais pesquisas na área. 


\section{Considerações finais}

O desenvolvimento de crianças e adolescentes é atravessado por diversos aspectos que necessitam ser considerados, como aqueles de cunho social, econômico, assim como o meio familiar no qual se está inserido e as práticas adotadas no mesmo. De tal maneira, é relevante elaborar formas de impactar positivamente o público mais jovem, visto que ao atuar com crianças e adolescentes, no desenvolvimento precoce de competências, espera-se estar atuando em atitudes e decisões que estes terão quando adultos.

Em tal perspectiva, é necessário um olhar direcionado aos estudos voltados para o ensino de HV, pois buscam impactar em escolhas mais saudáveis, proporcionando uma diminuição de comportamentos de risco, como o uso abusivo de substâncias psicoativas e o comportamento sexual não seguro. Assim sendo, intervenções em HV, principalmente de forma grupal, são pontuadas como de grande relevância por, dentre seus diversos benefícios, estimularem o contato e convívio social, em que no próprio grupo se torna possível praticar as habilidades discutidas.

No Brasil ainda não são muitos os estudos envolvendo intervenções em $\mathrm{HV}$ com crianças e adolescentes. Todavia, a partir da pesquisa exploratória aqui realizada, torna-se evidente os benefícios que tais intervenções proporcionaram, visto que, em todos os programas encontrados, foi possível observar aspectos positivos das experiências, sobretudo considerando dados qualitativos, a partir dos relatos dos participantes, assim como de cuidadores. Ademais, são apontados a manutenção das melhorias, mesmo em entrevistas de seguimento.

Conclui-se, de tal forma, que investimentos em intervenções que trabalhem as $\mathrm{HV}$ se fazem necessários e são relevantes, uma vez que podem ser inseridos em diversos contextos, versando sobre diferentes temáticas. Estas intervenções são benéficas, principalmente para o público infantojuvenil, dado a sua reverberação positiva ao desenvolvimento psicossocial, promovendo uma melhora na qualidade de vida como um todo. Salienta-se, conjuntamente a isso, a importância de se trabalhar essas habilidades para além do âmbito escolar, onde o foco tem se dado, ao se pensar em formas de atingir crianças e adolescentes em demais contextos.

\section{REFERÊNCIAS}

BOLSONI-SILVA, A. T. et al. Caracterização de programas de intervenção com crianças e/ou adolescentes. Arq. bras. psicol., Rio de Janeiro, v. 62, n. 1, p. 104-118, abr. 2010. Disponível em: http://pepsic.bvsalud.org/scielo.php?script=sci_arttext\&pid=S180952672010000100012\&lng=pt\&nrm=iso. Acesso em: 16 set. 2021. 
BOTVIN, G. J. Preventing Adolescent Drug Abuse through Life Skills Training: Theory, Evidence of Effectiveness, and Implementation Issues. In: CANE, J. Social Programs that work. New York: Russel Sage Fundation, 1998. p. 225-257.

BOTVIN, G. J.; GRIFFIN, K.W. Life Skills Training: Empirical Findings and Future Directions. The Journal of Primary Prevention, Cambridge, v. 25, n. 2, 2004, p. 211-232. Disponível em: https://link.springer.com/article/10.1023/B:JOPP.0000042391.58573.5b. Acesso em: 16 set. 2021.

CUNHA, N.; RODRIGUES, M. C. O desenvolvimento de competências psicossociais como fator de proteção ao desenvolvimento infantil. Est. Inter. Psicol., Londrina, v. 1, n. 2, p. 235 248, jun. 2010. Disponível em:

http://pepsic.bvsalud.org/scielo.php?script=sci_arttext\&pid=S2236$64072010000200008 \& \operatorname{lng}=$ pt\&nrm=iso. Acesso em: 16 set. 2021.

FRANCO, G. R. Habilidades de Vida: implementação e avaliação de uma pesquisaintervenção com adolescentes em situação de vulnerabilidade social. 2014. Dissertação (Mestrado em Psicologia) - Instituto de Ciências Humanas, Universidade Federal de Juiz de Fora, Juiz de Fora, 2014. Disponível em: https://repositorio.ufjf.br/jspui/handle/ufjf/800. Acesso em: 16 set. 2021.

FUNDAÇÃO OSWALDO CRUZ (FIOCRUZ). Instituto Nacional de Saúde da Mulher, da Criança e do Adolescente Fernandes Figueira. COVID-19 e Saúde da Criança e do Adolescente. Rio de Janeiro, 2020a. Disponível em:

http://www.iff.fiocruz.br/pdf/covid19_saude_crianca_adolescente.pdf. Acesso em: 16 set. 2021.

FUNDAÇÃO OSWALDO CRUZ (FIOCRUZ). Saúde Mental e Atenção Psicossocial na COVID-19: Crianças na Pandemia COVID-19. Rio de Janeiro, 2020b. Disponível em: https://www.fiocruzbrasilia.fiocruz.br/wpcontent/uploads/2020/05/crianc\%CC\%A7as_pandemia.pdf. Acesso em: 16 set. 2021.

GORAYEB, R.; NETTO, J. R. C.; BUGLIANI, M. A. P. Promoção de saúde na adolescência: Experiência com programas de ensino de habilidades de vida. In: TRINDADE, Z. A.; ANDRADE, A. N. (org.). Psicologia e saúde: um campo em construção. São Paulo: Casa do Psicólogo, 2003. p. 89-100.

MANGRULKAR, L.; WHITMAN, C. V.; POSNER, M. Enfoque de habilidades para la vida para un desarrollo saludable de niños y adolescentes. Washington: Organización Panamericana de la Salud, 2001.

MARYAM, E. et al. Effectiveness of life skills training on increasing self-esteem of high school students. Procedia - Social and Behavioral Sciences, v. 30, p. 1043-1047, 2011. DOI: $10.1016 /$ j.sbspro.2011.10.203

MINTO, E. C. Ensino de habilidades de vida para adolescentes vinculados a instituições profissionalizantes, no município de Ribeirão Preto/SP. 2005. Dissertação (Mestrado em Psicologia) - Faculdade de Filosofia, Ciências e Letras de Ribeirão Preto, Universidade de São Paulo, Ribeirão Preto. DOI: 10.11606/D.59.2006.tde-18052009-160841 
MINTO, E. C. et al. Ensino de habilidades de vida na escola: uma experiência com adolescentes. Psicologia em Estudo, v. 11, n. 3, p. 561-568, 2006. DOI: 10.1590/S141373722006000300012

MURTA, S. G. et al. Prevenção primária em saúde na adolescência: avaliação de um programa de habilidades de vida. Estudos de Psicologia, Natal, v. 14, n. 3, p. 181-189, 2009. DOI: 10.1590/S1413-294X2009000300001

MURTA, S. G. et al. Programa de habilidades interpessoais e direitos sexuais e reprodutivos para adolescentes: um relato de experiência. Psico-USF, v. 17, n. 1, p. 21-32, 2012. DOI: $10.1590 /$ S1413-82712012000100004

MURTA, S. G. et al. Prevenção à violência no namoro e promoção de habilidades de vida em adolescentes. Psicologia USP, v. 24, n. 2, p. 263-288, 2013. DOI: 10.1590/S010365642013000200005

MURTA, S. G.; DEL PRETTE, A.; DEL PRETTE, Z. A. P. Prevenção ao sexismo e ao heterosexismo entre adolescentes: contribuições do treinamento em habilidades de vida $\mathrm{e}$ habilidades sociais. Revista de Psicologia da Criança e do Adolescente, v. 1, n. 2, p. 73-85, 2010 .

NADALETI N. P. et al. Habilidades de vida: estratégia de prevenção do uso de substâncias psicoativas entre adolescentes. Adolesc Saude, 2018, v. 15, n. 3, p. 36-43. Disponível em: http://adolescenciaesaude.com/detalhe_artigo.asp?id=731\&idioma=Portugues. Acesso em: 16 set. 2021.

NEUFELD, C. B. et al. PRHAVIDA - Programa cognitivo-comportamental de habilidades de vida para crianças e adolescentes. In: NEUFELD, C. B. Intervenções e Pesquisas em Terapia Cognitivo-Comportamental com indivíduos e grupos. Novo Hamburgo: Sinopsys, 2014. p. 80-115.

PAIVA, F. S.; RODRIGUES, M. C. Habilidades de vida: uma estratégia preventiva ao consumo de substâncias psicoativas no contexto educativo. Estud. pesqui. psicol., Rio de Janeiro, v. 8, n. 3, dez. 2008. Disponível em:

http://pepsic.bvsalud.org/scielo.php?script=sci_arttext\&pid=S1808-

$42812008000300009 \& \operatorname{lng}=$ pt\&nrm=iso. Acesso em: 16 set. 2021.

RODRIGUES, M. C. et al. Prevenção e promoção de saúde na escola: concepções e práticas de psicólogos escolares. Gerais, Rev. Interinst. Psicol., Juiz de Fora, v. 1, n. 1, p. 67-78, jun. 2008. Disponível em: http://pepsic.bvsalud.org/scielo.php?script=sci_arttext\&pid=S1983$82202008000100008 \& \operatorname{lng}=$ pt\&nrm=iso. Acesso em: 16 set. 2021.

SUPARP, J. et al. Life Skills Development Program to Reduce Bullying and to Promote Good Practices among Primary School Students, Samut-Sakorn Province, Thailand. J Public Health, v. 40, n. 1, p. 7-16, 2010. Disponível em: https://he02.tci-

thaijo.org/index.php/jph/article/view/7876/6838. Acesso em: 16 set. 2021. 
UNITED NATIONS (UN). Module VII: Life Skills. 2006. Disponível em:

https://www.unodc.org/pdf/youthnet/action/message/escap_peers_07.pdf. Acesso em: 16 set. 2021.

ORGANIZAÇÃO MUNDIAL DA SAÚDE (OMS). Life skills education for children and adolescents in schools. Pt. 1, Introduction to life skills for psychosocial competence. Pt. 2, Guidelines to facilitate the development and implementation of life skills programmes. 2. rev. Geneva, Switzerland, 1997. Disponível em:

https://apps.who.int/iris/bitstream/handle/10665/63552/WHO_MNH_PSF_93.7A_Rev.2.pdf? sequence=1\&isAllowed=y. Acesso em: 16 set. 2021 .

ORGANIZAÇÃO MUNDIAL DA SAÚDE (OMS). Partners in life skills education. Geneva, Switzerland, 1999.

ORGANIZAÇÃO MUNDIAL DA SAÚDE (OMS). Skills for health: skills-based health education including life skills: an important component of a child-friendly/health-promoting school. Geneva, 2003. Disponível em: https://apps.who.int/iris/handle/10665/42818. Acesso em: 16 set. 2021.

\section{Como referenciar este artigo}

SANTOS, K. B.; CARVALHO, A. N. Intervenções em Habilidades de Vida: contribuições para o desenvolvimento psicossocial de crianças e adolescentes. Doxa: Rev. Bras. Psico. e Educ., Araraquara, v. 22, n. 00, e021015, 2021. e-ISSN: 2594-8385. DOI: https://doi.org/10.30715/doxa.v22i00.15349

Submetido em: 06/08/2021

Revisões requeridas em: 26/09/2021

Aprovado em: $14 / 10 / 2021$

Publicado em: 01/10/2021 